\title{
similitude et essais sur modèles réduits : validité de la modélisation
}

\author{
similarity and model test : \\ validity of the modelization
}

\author{
P. HABIB \\ Laboratoire de mécanique des solides* \\ (E.P. - ENSM Paris - ENPC - Associé au CNRS)
}

Rev. Franç. Géotech. n 48 , pp. 35-41 (juillet 1989)

\section{Résumé}

Les conditions de validité des essais sur modèles réduits en mécanique des sols sont étudiées, d'une part pour la représentation de la structure et de son chargement, et d'autre part, pour la représentation du comportement des matériaux. Les effets d'échelle sont particulièrement étudiés et plus spécialement ceux qui sont liés à l'apparition d'une surface de glissement pendant la rupture du modèle réduit.

\footnotetext{
Abstract

Validity conditions for model tests in soil mechanics are studied for structure representation and for loading representation on one hand, for constitutive laws of the materials on the other hand. Scale effects are particularly examined and specially those connected with slip surface formation during the model failure.
} 


\section{INTRODUCTION}

La résolution d'un problème de mécanique pour les applications de l'art de l'ingénieur à l'étude des structures du génie civil se limite le plus souvent à la détermination de la charge de rupture de la structure étudiée, des déformations à la rupture, de la charge de service, des déformations de service.

II faut parfois déterminer davantage de choses, comme les périodes propres des vibrations de la structure et leur amortissement, ou bien on a besoin de connaître des éléments très précis comme le champ des contraintes en tous points, mais les résultats précédents sont généralement suffisants.

Dans ces conditions, l'ingénieur a besoin pour poser correctement le problème de mécanique correspondant :

- de la définition de la structure

- de ses conditions aux limites:

- de son mode de chargement ;

- et enfin des relations de comportement de la matière, ou des matières, dont est composée la structure.

Le recours aux méthodes analogiques, essentiellement en similitude par des modèles réduits, consiste à reproduire la structure et ses conditions aux limites, le système de chargement et les lois de comportement des matériaux du prototype, bien entendu en respectant les lois mathématiques et physiques correspondant à ce qu'il est convenu d'appeler les conditions de similitude. Elles sont actuellement bien connues [5], [10], [13], [15] et il n'en sera pas fait mention ici, car il n'y a pas pour l'instant de problèmes majeurs sur ce sujet.

\section{REPRÉSENTATION DE LA STRUCTURE ET DE SON CHARGEMENT}

La réalisation d'un modèle réduit de la structure est, en général, assez simple. C'est même de là que vient lattirance des ingénieurs pour les maquettes. La construction d'un modèle à une échelle géométrique courante est un travail facile. Il peut y avoir cependant quelques difficultés pour des cas singuliers et, par exemple, pour la reproduction de certaine technologie de fabrication: soudure par points entre deux tôles, scellement par injection d'un ancrage dans le sol, présence d'un film de peinture sur une tôle mince, etc. mais, dans la plupart des cas, quelle que soit l'échelle, la réalisation géométrique ne pose pas de problèmes insurmontables.

On peut en dire de même des conditions aux limites : la restitution des conditions correctes représentant un encastrement, une articulation, une liaison avec frottement unilatéral, un contact lisse, etc., ne pose pas de question au niveau des principes, mais la réalisation peut demander du temps et du soin. Par exemple, la figure 1, d'après Maury [11], représente le chargement à la surface d'un massif rocheux stratifié, avec un frottement nul entre les strates, sur un modèle photoélastique : il a fallu un mois de travail pour pouvoir prendre cette photographie. Il s'agissait d'un modèle difficile, car avec $\varphi=0$, l'empilement était très instable; mais, il a fallu obtenir des contacts sans irrégularité, modifier le cadre, corriger le chargement de façon à symétriser parfaitement les isochromatiques, etc. Dans un modèle réduit à la rupture, on ne visualise pas le champ des contraintes : ce n'est pas une raison pour ne pas traiter avec autant de soin la réalisation matérielle de la maquette. Un problème de mécanique est généralement très sensible aux conditions aux limites et il n'est pas suffisant de dire que les conditions aux limites du modèle étant aussi mal définies que celles du prototype, on peut considérer comme satisfaisante la solution obtenue!

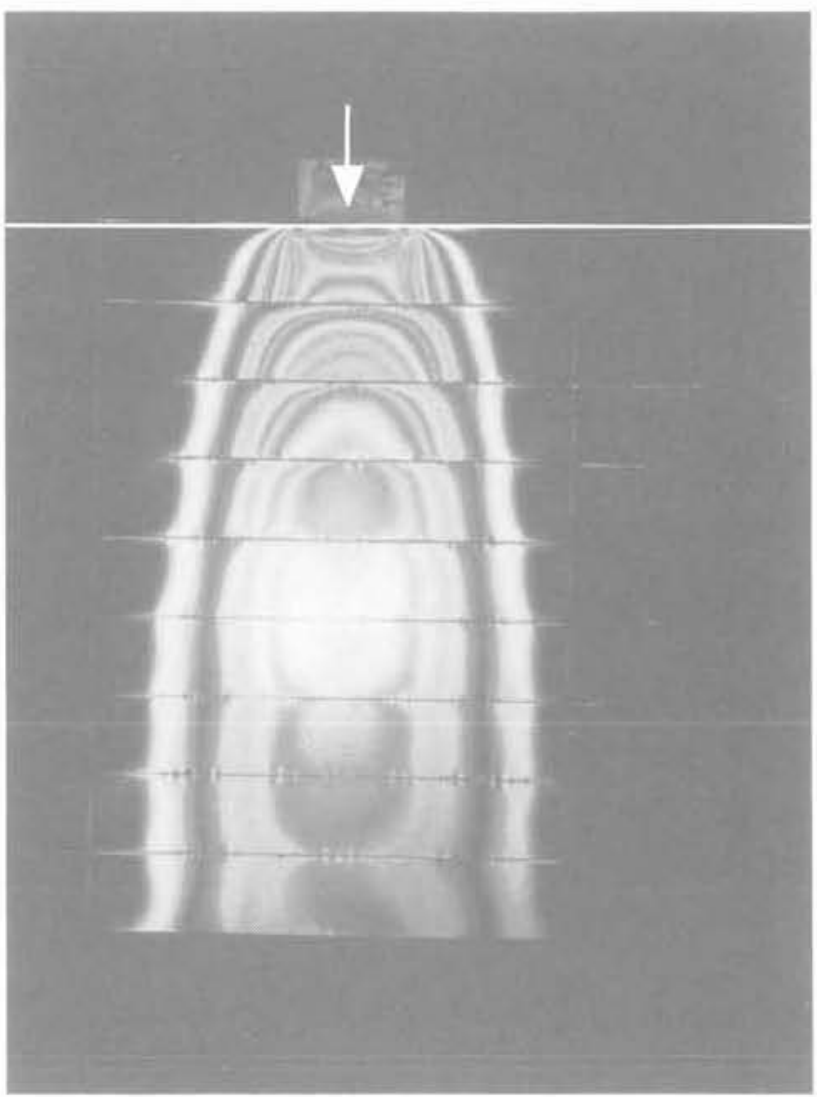

Fig. 1. - Fondation à la surface d'un massif stratifié avec frottement faible aux interfaces, [11].

Fig. 1. - Shallow foundation on a layered model with small interfacial friction, [11]

Pour les conditions initiales, il faut insister sur deux difficultés. L'une porte sur les conditions aux limites en déplacement ; bien entendu, il est possible de disjoindre l'échelle des déplacements $\dot{\Delta} \ell$ de l'échelle des longueurs ' $\ell$; mais lorsqu'on mène le modèle à la rupture et qu'on souhaite conserver la géométrie des déformations, il faut avoir $\Delta \ell=\ddot{\ell}$; pour des échelles des longueurs petites, cette condition peut être difficile à assurer avec précision. Par exemple, sous une fondation, un déplacement de $1 \mathrm{~cm}$ n'est pas négli- 
geable au point de vue de son effet sur la distribution des contraintes; avec un modèle réduit, dont l'échelle des longueurs est $1 / 100$, cela signifie une précision de $1 / 10 \mathrm{~mm}$, ce qui est bien difficile à atteindre partout. De la même façon, la réalisation d'un modèle en déformation plane dans une cuve à face parallèle est beaucoup plus difficile qu'on ne le croit généralement, car il est très difficile d'assurer $\epsilon_{2}=0$ avec une précision sur $\Delta \ell_{2}$ de l'ordre du $1 / 10 \mathrm{~mm}$ pendant tout le chargement.

La deuxième difficulté porte sur les incertitudes qui peuvent régner au niveau de l'état initial des contraintes, non pas à cause de la difficulté de la restitution de ces contraintes, mais bien parce que le champ des contraintes initiales dans une structure n'est pas toujours connu avec toute la précision souhaitable : la distribution des contraintes initiales dues au poids propre sous la fondation d'un ouvrage est-elle du type Boussinesq, ou bien est-ce une pression uniforme? Quel est l'état initial des contraintes sur le revêtement d'une galerie? Le frottement latéral initial sur un pieu battu? Les contraintes initiales dans une pièce sujette à un retrait d'origine thermique ou physique? Souvent cette incertitude n'a pas trop de conséquences, notamment lorsque les matériaux dont on étudie la rupture obéissent à un critère de Tresca, car dans un tel cas, la charge ultime n'est pas altérée, seules les déformations pour y parvenir peuvent être modifiées. Dans d'autres cas, pour les matériaux qui répondent au critère de Coulomb, cela peut ne pas être vrai et cela nécessite un examen attentif. C'est par exemple le cas de la pression d'injection du scellement d'un ancrage dans un sol sableux, qui a une influence reconnue sur la résistance à l'arrachement [6]. On a des résultats analogues pour le frottement latéral des pieux.

La représentation du chargement ne présente, en général, pas de difficultés majeures et il suffit de conserver les mêmes conceptions que pour la simulation de la forme. Dans certains cas, pour des problèmes de dynamique, lorsque l'échelle du temps est petite, la représentation correcte de la chronologie de la sollicitation peut être un peu délicate, et même être à lorigine de difficultés technologiques sérieuses : c'est, par exemple, ce qui se produit pour la modélisation d'une sollicitation sismique, et s'il faut reproduire correctement un spectre contenant des vibrations situées entre $1 \mathrm{~Hz}$ et $1 / 10 \mathrm{~Hz}$ avec une échelle du temps de $1 / 100$, on doit fabriquer des spectres de vibration compris entre $1 / 100$ et $1 / 1000 \mathrm{~Hz}$. Différentes méthodes ont été proposées. Ainsi, par exemple, A. ZÉLIKSON, au LMS, et LEGUAY, au CESTA, ont utilisé à cet effet des rafales de tirs d'explosifs ou de tubes à choc au voisinage de cavités, jouant le rôle de résonateurs de Helmholtz.

Remarquons que les solutions qui ont été proposées donnent souvent des résultats dont la fidélité est plus grande que celle de la nature : il n'est pas évident, par exemple, que la sollicitation sismique du séisme d'El Centro se reproduise à nouveau à l'identique, même si les contenus fréquentiels des séismes de la région sont analogues. Par contre, sur un modèle réduit, on reproduira vingt fois le même tremblement de terre.

\section{REPRÉSENTATION DU COMPORTEMENT DES MATÉRIAUX}

Dans la simulation sur maquette, ce qui est le plus délicat, c'est la représentation correcte du comportement des matériaux. En élasticité, dans le domaine linéaire, même pour des problèmes compliqués, cela est facile et les résultats sont excellents. Ainsi, dans le domaine des petites déformations, les résultats obtenus au LMS, avec l'Institut Français du Pétrole, pour examiner le comportement cyclique sous tempête de la fondation d'une plate-forme à embase-poids en Mer du Nord, ont été remarquablement confirmés par les observations in situ (déplacement cumulé de l'ordre de $0.5 \mathrm{~m})$.

M.P. LUONG, au LMS, a étudié [8] les non-linéarités des vibrations des pieux et des groupes de pieux dans le sol. Plus récemment, il a étudié, par la même méthode, des fondations précontraintes [9] pour des pylônes électriques, et les résultats obtenus ont été très bien confirmés par des essais in situ en vraie grandeur effectués avec EDF. Des travaux remarquables sur les petites déformations, qui mènent à la liquéfaction des sables, ont été faits par le Professeur A. SCHOFIELD à Cambridge pour des barrages en terre [7].

Si l'utilisation des matériaux équivalents pour représenter les déformations élastiques est largement développée malgré quelques difficultés et, par exemple, la nécessité de garder le même coefficient de Poisson entre la maquette et le prototype lorsque le modèle n'est pas simplement connexe, par contre, dès qu'on veut s'approcher de la rupture, le nombre de paramètres nécessaires pour représenter correctement le comportement du matériau devient très grand et il n'est souvent pas possible de trouver un matériau dont toutes les propriétés soient en similitude avec le réel : en plus des coefficients définissant le critère de rupture, $\phi$ et $C$ pour un critère de Coulomb, mais peut-être d'autres coefficients si la courbe intrinsèque a une allure parabolique, il y a en plus les coefficients d'élasticité linéaire ou non-linéaire et de plasticité (écrouissage), la dilatance, la densité critique, la viscosité plastique, la perméabilité, etc. Les choix sont, la plupart du temps, suffisamment nombreux et difficiles pour qu'il soit pratiquement impossible de les satisfaire tous. Il est bien connu que l'on peut tenter alors d'utiliser pour la maquette, le même matériau que celui du prototype et sous les mêmes contraintes. L'échelle des contraintes étant alors égale à l'unité, il résulte des équations d'équilibre que l'échelle des accélérations doit être égale à linverse de l'échelle des longueurs. Lorsque le rôle du poids propre est important, on est dans le domaine d'élection des essais en centrifugeuse. Mais, ce n'est pas toujours suffisant et on peut être amené à utiliser à la fois centrifugeuse et matériaux équivalents, par exemple, pour des problèmes géologiques. Ainsi, A. ZÉLIKSON au LMS [16], pour représenter la montée d'un dôme de sel à partir d'une couche sédimentaire de sel a utilisé une échelle des longueurs de $10^{-5}$. PELTZER et TAPPONIER [12] pour représenter la collision IndeAsie, ont utilisé une échelle des longueurs de $10^{-7}$; avec une échelle des accélérations de 100 , la rela- 
tion $\sigma=\rho \dot{g} \ell$ donne dans ce deuxième exemple, puisque $\dot{\rho}$ reste voisin de l'unité, une échelle des contraintes $\dot{\sigma}$ de l'ordre $10^{-5}$. Si le module d'élasticité de la roche est de $50000 \mathrm{MPa}$, celui de la maquette sera de $0,5 \mathrm{MPa}$. Si la résistance à la compression de la roche est $100 \mathrm{MPa}$, celle de la matière dont est fait la maquette sera de $1 \mathrm{kPa}$. On conçoit que bien des choses doivent manquer dans une telle représentation, et l'intérêt des résultats obtenus est lié au fait que les caractéristiques mécaniques à long terme d'une plaque continentale sont elles-mêmes très mal connues.

L'emploi du matériau du prototype pour fabriquer le modèle réduit est donc particulièrement intéressant y compris pour des problèmes dynamiques, comme la pénétration d'un projectile dans de l'argile, tel que cela a été étudié au CESTA par LEGUAY et ZÉLIKSON. Mais, ce n'est pas toujours possible, par exemple parce que le matériau n'existe pas : voici le cas de la vidange d'un silo métallique ; on veut représenter une tôle de $3 \mathrm{~mm}$ d'épaisseur à l'échelle du $1 / 20$, or la tôle de 1,5/10 mm d'épaisseur du même métal est beaucoup plus écrouie que la tôle de $3 \mathrm{~mm}$ et sa limite élastique est probablement beaucoup plus élevée. Et puis, il existe aussi un certain nombre de matériaux pour lesquels les propriétés mécaniques d'un grand morceau sont différentes de celles d'un petit morceau, et il devient difficile de comparer ce qui ce passe sur un modèle réduit avec ce qui se passe avec le prototype. On dit alors qu'il y a effet d'échelle. Ce terme recouvre le plus souvent des effets incompris ou tout au moins mal identifiés.

Pour les roches et les bétons, par exemple, il est bien connu que la résistance et la dispersion des résultats sont des fonctions décroissantes de la dimension du corps d'épreuve. L'origine de ces deux phénomènes est liée à la fissuration de la matière, le champ fissural et la distribution aléatoire des fissures pouvant d'ailleurs exister à différentes échelles: cristal, agrégat, bloc ou massif. Mais, il existe des cas dont l'interprétation est plus difficile. C'est par exemple celui des sables. La figure 2, issue de [3] et complétée, donne, en coordonnées doublement logarithmiques, des résultats d'essais de traction horizontale pour deux modèles d'ancres de poids différents sur du sable. Avec des matériaux pulvérulents, la force devrait être fonction du cube des longueurs, c'est-à-dire proportionnelle au poids de l'ancre. Dans la représentation de la figure 2 , les courbes représentatives devraient donc être des droites à $45^{\circ}$. On voit qu'il n'en est rien pour les deux modèles étudiés. Dans le cas présent, les essais sur modèle, effectués au LMS, avaient pour but d'optimiser en gravité normale une forme d'ancre. A partir des résultats expérimentaux, nous avons estimé que l'extrapolation des essais sur maquette ne pouvait être faite que par pas de 5 sur les forces, c'està-dire par pas de $\sqrt[3]{5}=1,71$ sur les longueurs. On avait prévu ainsi d'aller jusqu'à une force horizontale de $800 \mathrm{kN}$. Il est possible que cet effet d'échelle soit lié à une hétérogénéité des sables marins in situ, le matériau étant de plus en plus résistant en profondeur et donc, en fait, de moins en moins pénétré, relativement, par les grosses ancres. Il faut préciser qu'avec le même modèle d'ancre, dans de l'argile homogène, des résultats excellents au point de vue de la similitude ont été obtenus, et ont permis une extrapolation de 10 sur la longueur, soit 1000 sur la force, entre la maquette et le prototype in situ.
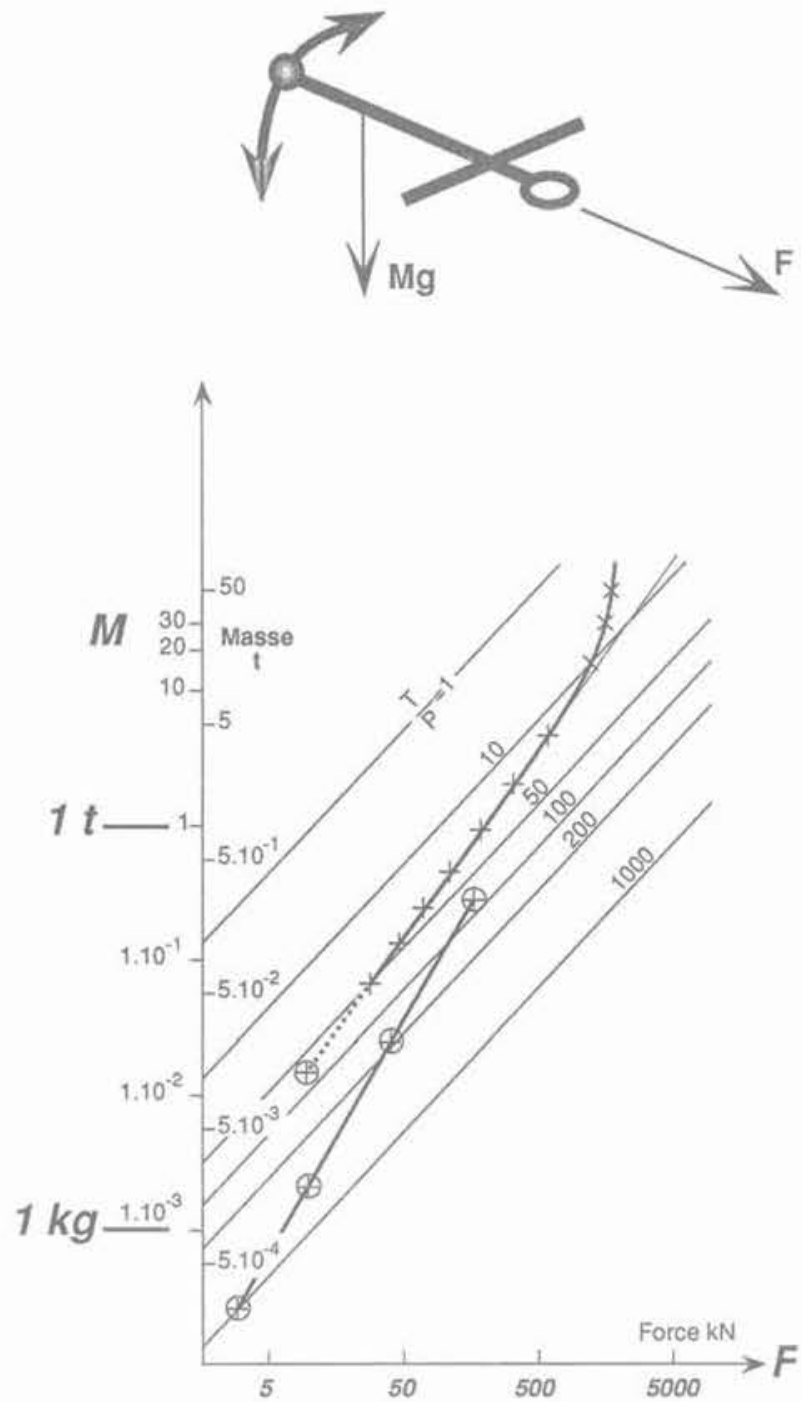

Fig. 2. - Effet d'échelle pour deux types d'ancres marines sur du sable, [3].

Fig. 2. - Scale effect for two marine anchors on sand, (3).

Des effets d'échelles sur les sables ont été obtenus par des expérimentateurs pour d'autres modèles. La figure 3, d'après TCHENG et ISEUX [14], indique des résultats obtenus avec des petits modèles de fondations superficielles sur un sable sec. Les essais de poinçonnement ont fait l'objet de nombreuses communications dans de nombreux Congrès Internationaux de Mécanique des Sols, et les tentatives d'interprétation sont variées ; tout se passe comme si un massif de sable, en laboratoire ou dans la nature, n'était pas homogène, comme si la couche superficielle était de meilleure qualité : pour être plus précis, dans la mesure où la résistance au poinçonnement peut être donnée par une expression de la forme $q_{u}=1 / 2 \gamma B N_{i}(\phi)$ on constate que les plus petits poinçons donnent les plus grandes valeurs 


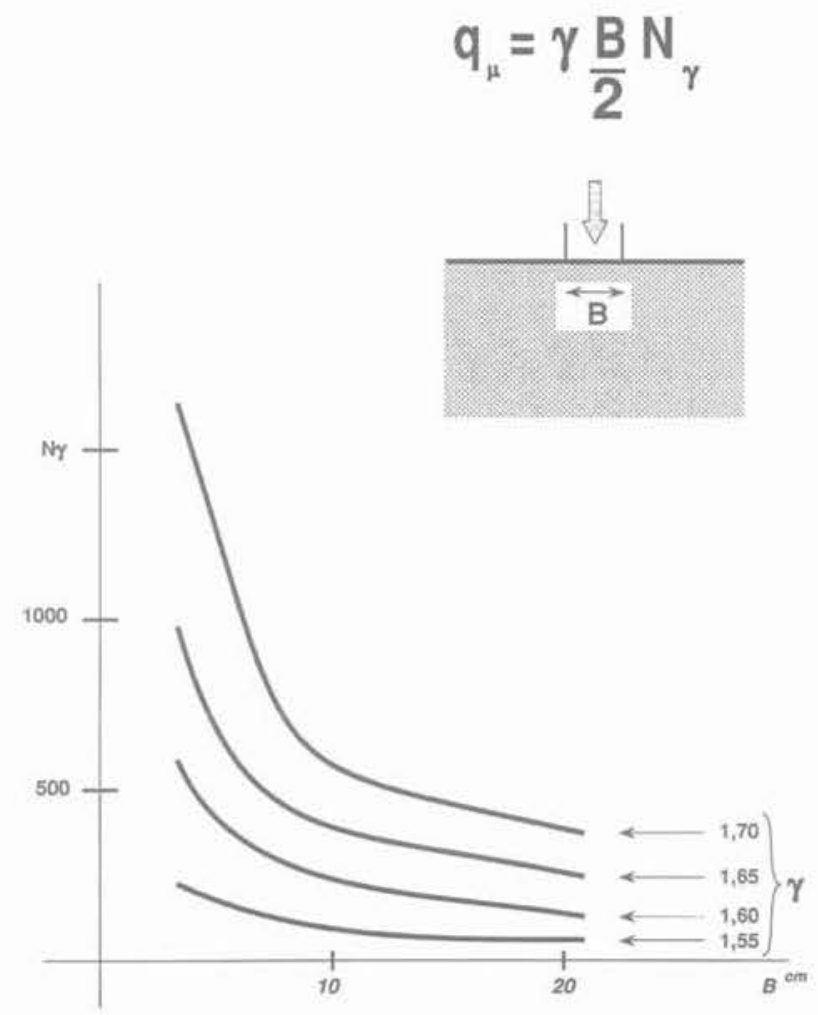

Fig. 3. - Effet d'échelle pour des fondations superficielles sur du sable, [14].

Fig. 3. - Scale effect for shallow foundations on sand, [14].

de $\mathrm{N}_{\gamma}$; on pourrait en conclure que l'angle de frottement interne $\phi$ du sable (ou que la densité du sable) est plus grand en surface qu'en profondeur. Cela parait peu vraisemblable, en tout cas aucune mesure n'a pu le confirmer.

Une interprétation de ce phénomène a été proposée [2], [4], liée à la dilatance des sables et au phénomène corrélatif du radoucissement de la courbe effortdéformation observé dans les sables denses. On peut la résumer comme suit. Une surface de glissement se forme après un déplacement limité ; dans un champ de déformation hétérogène, comme celui qui existe sous une fondation ou auprès d'un mur de soutènement, la surface de glissement commence à apparaître au bord de la fondation ou au coin du mur qui poinçonne le sol lorsque le déplacement atteint un certain seuil, de l'ordre de 3 à $4 \mathrm{~mm}$, puis elle se développe jusqu'à émerger à la surface libre du massif. Pour un mur de soutènement assez grand, B. DUTHILLEUL [1] a observé que la force sur le mur était proportionnelle à la longueur de la ligne de glissement en formation. Pour un tel exemple, avec un sable dense, on a indiqué sur la figure 4 la zone de dilatance active située autour du front de la surface de glissement en formation, un peu en avant et un peu en arrière; mais, près du mur, là ou la surface de glissement est plus « âgée », la dilatance dans la surface de glissement a épuisé les réserves de déformation : la rupture est progressive et la courbe "forcedéplacement " ne présente pas de maximum alors que le comportement du matériau en présente un. $\mathrm{Si}$ l'on imagine maintenant un petit modèle dont la longueur soit égale, ou inférieure, à celle de la zone de dilatance active (fig. 4), on voit que pour ce modèle la rupture va cumuler l'effet de frottement et celui de la dilatance, qui était en quelque sorte " diluée " dans le grand modèle. Il en résulte que la résistance du petit modèle est, proportionnellement, plus grande que celle du grand modèle et que le comportement du petit modèle, c'est-à-dire la courbe * force-déplacement *, présente du radoucissement, comme le comportement du sable dense. Il y a effet d'échelle. On peut montrer que cet effet est en relation avec la dimension des grains. Sur la figure 5 , on a représenté deux groupes de matériaux pulvérulents, qui sont strictement homothétiques. Il en résulte que leurs angles de frottement interne $\phi_{1}$ et $\phi_{2}$ sont identiques, ainsi que leurs angles de dilatance $\Psi_{1}$ et $\Psi_{2}$. Mais, la dilatance normale $\delta$ dans le plan de glissement est plus grande pour le matériau grossier que pour celui dont les grains sont les plus petits, et l'on a $\delta_{2}=\dot{\ell} \delta_{1}$, où $\dot{\ell}$ est le rapport d'homothétie. Cette conclusion serait évidemment inchangée si la surface de glissement intéressait une certaine épaisseur de sable, quelques couches de grains, au lieu de n'intéresser comme sur la figure 5 que le désenchevêtrement d'un seul contact de grains. Il est très probable qu'un effet d'échelle lié à la rupture progressive existe aussi pour les argiles raides radoucissantes, même si les expériences manquent sur ce sujet. De toutes façons, même si l'existence d'un radoucissement du comportement ne provoque pas obligatoirement le phénomène de localisation, la conjonction " radoucissement-effet d'échelle * a été signalée déjà par plusieurs auteurs.

Pour un modèle réduit en centrifugeuse avec un sable dense, les phénomènes évoqués ci-dessus doivent se retrouver intégralement, surtout si l'échelle des contraintes est égale à l'unité. L'effet d'échelle sur le petit modèle peut conduire alors à des résultats trop forts (c'est-à-dire à des résistances trop grandes), et le modèle paraît alors optimiste. Il est donc nécessaire, pour les essais en modèles réduits, qu'il ne se produise pas de surface de glissement et que les problèmes étudiés correspondent à des cas, où, en fonction des conditions aux limites et du comportement élasto-plastique des matériaux, il n'apparaít pas de localisation de la déformation, et où le milieu reste continu. Dans le cas contraire, il faut être très prudent pour tirer des modèles réduits des conclusions autres que qualitatives.

\section{CONCLUSIONS}

Les essais sur modèles réduits donnent, dans de nombreux cas, des résultats extrêmement intéressants en mécanique des sols, et les centrifugeuses, qui permettent une similitude particulièrement sophistiquée, sont dans certains cas véritablement indispensables. Des précautions ont été indiquées pour la construction des maquettes et elles doivent être respectées. En particulier, il faut faire très attention aux effets d'échelle, et notamment éviter ceux qui sont liés à la localisation des déformations et à la formation de surface de glissement dans des processus de rupture progressive. 


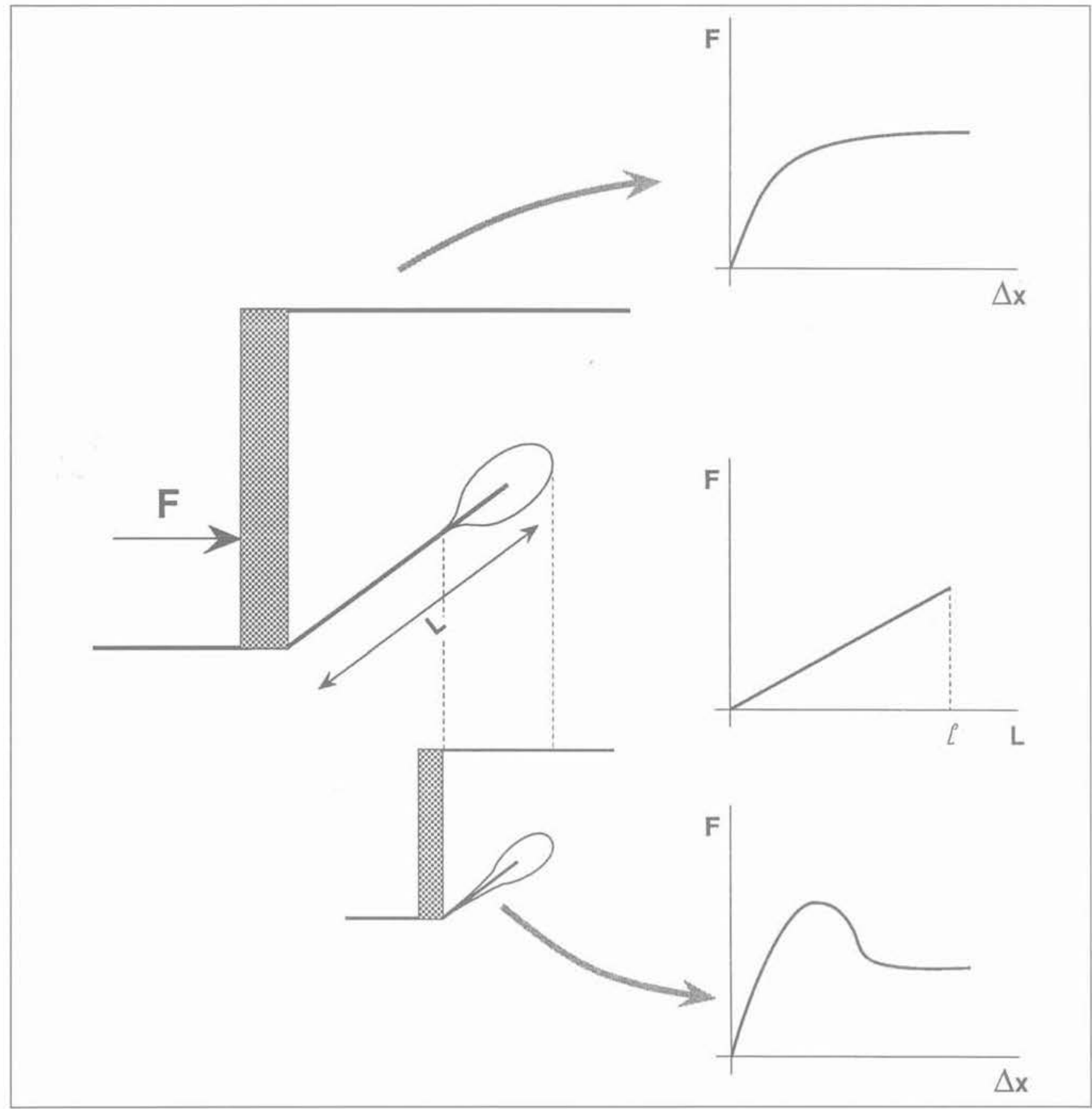

Fig. 4. - Effet d'échelle pour un mur de soutènement.

Fig. 4. - Scale effect for a retaining wall. 


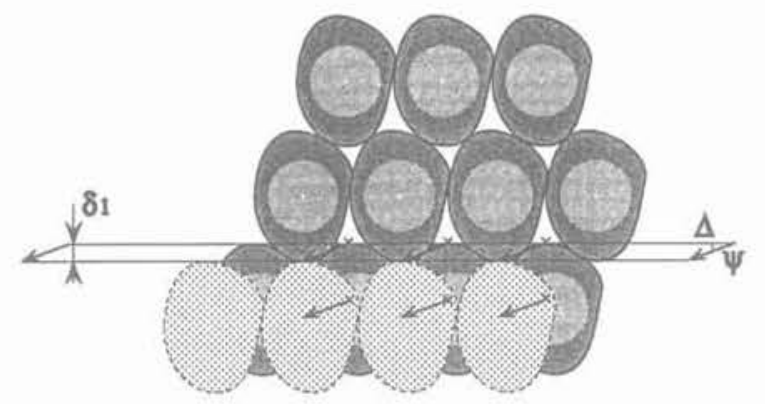

(1)

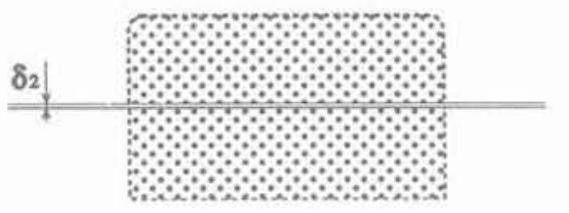

(2)

Fig. 5. - Effet d'échelle : aspect microscopique de la surface de glissement dans un sable dense.

Fig. 5. - Scale effect : microscopical aspect of the slip surface in a dense sand.

\section{BIBLIOGRAPHIE}

[1] DUTHILLEUL B. (1983), « Rupture progressive, simulation physique et numérique ». Thèse de Doct-Ing., USMG, INPG Grenoble.

[2] HABIB P. (1984), \& Scale effect for shallow footing on dense sand ». Proc. ASCE, J. of the Geotechnical Eng. Div., vol. 100, $n^{\circ}$ GT1, Janvier 1974, pp. 95-99.

[3] HABIB P. (1980), « Ancres marines-Ancrages à terre ".. Revue Française de Géotechnique, $\mathrm{n}^{\circ} 11$, Mai 1980, pp. 17-28.
[4] HABIB P. (1985), "Effet d'échelle et surfaces de glissement ». Revue Française de Géotechnique, $n^{\circ} 31$ (2e trimestre 1985).

[5] HABIB P., ZÉLIKSON A. (1985), « Modèles réduits en dynamique ». Génie Parasismique, Presses ENPC, Paris, pp. 563-568.

[6] JORGE G.R. (1969), «Le tirant IRP réinjecta. ble spécial pour terrains meubles ». 7e Congrès Int. SIMSTF, Mexico, Août 1969, Session Spéciale 15, p. 162.

[7] LEE F.H., SCHOFIELD A.N. (1988), * Centrifuge modelling of sand embankments and islands in earthquakes ». Géotechnique 38, $n^{\circ} 1$ (March 1988), pp. 45-58.

[8] LUONG M.P. (1986), «Centrifugal models of piles and pile groups under lateral harmonic excitations and seismic actions ». In Numerical Methods in Offshore Piling, Ed. Technip, pp. 511-525.

[9] LUONG M.P., MARTIN A., de PARNY R. (1988), «Fondations précontraintes pour supports aériens ». In Supports Aériens : Choix Optimisation - Essais, ESE, Gif-sur-Yvette, pp. 59-66.

[10] MANDEL J. (1962), « Essais sur modèles réduits en mécanique des terrains. Etude des conditions de similitude ». Revue de l'Industrie Minérale, Sept. 1962, vol 44.

[11] MAURY V. (1970), « Mécanique des milieux stratifiés ». Dunod, Paris, 1970, pp. 98 et 104.

[12] PELTZER G., TAPPONIER P. (1988), « Formation and evolution of srike-slip faults, rifts and basin during the India-Asia collision : an experimental approach». (A paraître dans Journal of Geophysical Research).

[13] SAINT-GUILHEM R. (1985), * Sur les fondements de la similitude physique : le théorème de Federman ». Journal de Mécanique Théorique et Appliquée, vol. 4, n³, pp. 337-356.

[14] TCHENG Y., ISEUX J. (1966), * Nouvelles recherches sur le pouvoir portant des milieux pulvérulents : fondations superficielles et semiprofondes ». Annales ITBTP, $\mathrm{n}^{\circ} 227$, Paris, Nov. 1986, pp. 1267-1282.

[15] WEBER J.D., "Les applications de la similitude physique aux problèmes de la Mécanique des Sols ». Ed. Eyrolles, Gauthier-Villars, Paris, 1971.

[16] ZÉLIKSON A. (1988), * The use of large centrifuges for modeling geological instabilities caused by gravity anomalities », In "Centrifuge 88 », Paris. 